\title{
Inviscid Interaction of Vortex Rings: Approach to Singularity?
}

\author{
John R. Grant \\ Bldg. 1302, Code 8233 \\ Naval Undersea Warfare Center \\ Newport, RI 02841 USA \\ Jeffrey S. Marshall \\ Department of Mechanical Engineering \& Institute of Hydraulic Research \\ The University of Iowa \\ Iowa City, IA 52242 USA
}

\begin{abstract}
The interaction of two vortex rings in an inviscid fluid is investigated numerically using a Lagrangian vorticity method. This method uses interpolation based on Delaunay triangulation of the computational points. The calculational results indicate that this method is well-suited to the computation of these and similar cases. In particular, one of the most useful features of the method in this context is the ability to refine the resolution of the flow as vorticity is concentrated onto increasingly small scales. Such concentration, along with increase in the maximum norm of the vorticity, is a prominent feature of the computed interaction. Two cases are presented. Analysis of the results from one case shows that through the first $80 \%$ of the interaction, the growth in the maximum norm is approximately exponential. Later the flow appears to undergo a transition to a new regime not inconsistent with a growth proportional to $1 /\left(\mathrm{t}_{\mathrm{c}}-\mathrm{t}\right)$. However, the present calculations are not sufficiently long to confirm this behavior.
\end{abstract}

\section{Introduction}

In viscous fluids, reconnection of lines of vorticity is a commonly observed feature of the interaction of vortices. The ensuing complex and subtle flow has been studied in a variety of contexts [e.g., 1 and the references therein]. The reconnection process proceeds through the action of the viscosity of the fluid, and without viscosity, reconnection cannot take place, as stated by the Helmholtz vortex theorem [2].

Consequently, the interaction of vortices in an inviscid fluid will be strikingly different than when viscosity is present. Without viscosity to reconnect vortex lines or relieve regions of intensifying vorticity, there is a potential for concentrated regions of strong vorticity to appear and continue to develop. In particular, even a small amount of viscosity is thought to rule out the emergence of a singularity in the flow in finite time [3], and zero viscosity is generally thought to be a requirement for the appearance of a finite time singularity.

Thus time-dependent inviscid flows (governed by the three-dimensional Euler equations) where locally high vorticity may emerge and develop at small scales present a challenging topic for study. Nevertheless, analytical investigations have revealed constraints on the growth of vorticity [4 - 6] in these solutions, and at least one analytical model of an initially symmetric flow approaching finite time singularity has been derived [7]. Numerical solution of the Euler equations from initial conditions enables a wider range of flow situations to be considered. However, as implied by the preceding discussion, the necessity for the numerical method to be free of artificial viscosity is stringent. Kerr [8] describes calculations of the interaction of two tubular vortices using a Chebyshev method which apparently is inviscid. By setting up an initial grid of points clustered in the anticipated vicinity of the interaction and using symmetry, he is able to follow the interaction to the point where the approach to a finite-time singularity is plausible. A numerical strategy not employing such specialization is to use a series of calculations with decreasing viscosity to infer the trend toward inviscid behavior [9 - 11]. However, the range of viscosity that can be used is relatively small, and the extrapolation of viscous solutions to the inviscid case is not without ambiguity. 
Lagrangian methods, where the calculational points are transported by the computed velocity at the respective points, are recognized to be effectively inviscid. These methods enjoy several advantages for computing low- or zero-viscosity time-dependent vorticity dominated flow. First, since the initial points may be placed as desired, filtering [8]or other special treatments are not needed to specify the initial condition. Second, as the vorticity develops on increasingly small scales, the resolution of the flow by a grid-based method eventually will become insufficient. While additional points may be inserted adaptively, this is particularly difficult for Galerkin techniques. Lagrangian points, already irregularly spaced, allow new points to be added without regard to ordering, to maintain resolution. Maintenance of resolution is essential for following the flow evolution to times near the emergence of a singularity. Finally, with the exception of Galerkin techniques such as Fourier and Chebyshev methods, many gridbased methods are well-known to possess artificial viscosity [e.g., 12].

We have examined the inviscid interaction of two vortex rings using a Lagrangian method which does not employ the blob algorithm for integrating the Biot-Savart integral, and which is effective when regions of high vorticity are at the boundaries of the rotational flow (such as in the present case or flow past a body). The full algorithm for unbounded flow is outlined in the next section; discussion of the elaboration to bounded viscous flow will be presented elsewhere. Since one motive for performing these calculations is the evaluation of the overall method, the code is used in a general form -- no particular specialization to the case at hand is made. In particular, symmetry is not invoked to omit the calculation of one of the two rings. Thus, because the rings are initially symmetric and should remain so in an ideal case, differences in the rings during the calculation provide error estimates for the calculated results, aiding in the assessment of the method.

The computational results are presented and discussed in Section 2. The results indicate that the method is indeed inviscid or, at least, posesses only very low artificial viscosity. Finally, the computed behavior is discussed in terms of approach to a finite-time singularity.

\section{Numerical Formulation}

The Euler equations in vorticity form describe the evolution of inviscid fluid of uniform density

$$
\begin{gathered}
\frac{\partial \vec{\omega}}{\partial \mathrm{t}}+\overrightarrow{\mathbf{u}} \cdot \nabla \vec{\omega}=\vec{\omega} \cdot \nabla \overrightarrow{\mathbf{u}} \\
\nabla \cdot \overrightarrow{\mathbf{u}}=0
\end{gathered}
$$

where $\mathbf{u}$ is the velocity and $\omega$ is the vorticity,

$$
\vec{\omega}=\nabla \times \overrightarrow{\mathbf{u}} .
$$

With this relation and (1b), for unbounded flow we have the Biot-Savart integral

$$
\overrightarrow{\mathbf{u}}(\overrightarrow{\mathbf{x}})=\frac{1}{4 \pi} \int_{\mathrm{v}} \frac{\vec{\omega}\left(\overrightarrow{\mathbf{x}^{\prime}}\right) \times\left(\overrightarrow{\mathbf{x}}-\overrightarrow{\mathbf{x}^{\prime}}\right)}{\left|\overrightarrow{\mathbf{x}}-\overrightarrow{\mathbf{x}^{\prime}}\right|^{3}} \mathrm{~d}^{3} \mathrm{x}^{\prime}
$$

where $\mathrm{V}$ is the volume of fluid where the vorticity is non-zero. 
For the numerical solution of (1) and (2), the material derivative in (1a) is accounted for by computing the evolution of vorticity on points $\left(\mathbf{x}_{\mathrm{n}} ; \mathrm{n}=1, \mathrm{~N}\right)$, where $\mathrm{N}$ is the number of points, which are transported by the local velocity:

$$
\frac{\mathrm{d} \overrightarrow{\mathbf{x}}_{\mathbf{n}}}{\mathrm{dt}}=\overrightarrow{\mathbf{u}}\left(\overrightarrow{\mathbf{x}}_{\mathbf{n}}\right), \quad \mathrm{n}=1, \mathrm{~N}
$$

Equations (1a) and (3) are advanced in time using an Adams-Bashforth algorithm. The velocity required in both of these equations is computed at each time step with (2) using the current vorticity.

\section{Computation of the Biot-Savart integral}

The interpolation needed to numerically evaluate the Biot-Savart integral (2) given the vorticity field on a set of points is performed with the aid of a Delaunay triangulation [e.g., 13, 14] of the set of points. The most important features of a Delaunay triangulation for this use are that it may be performed quickly compared to other numerical operations during the calculation, that it produces tetrahedra connecting the computational points such that no tetrahdedron contains within itself a computational point, and that the subtended angles at the vertices of the tetrahedra are (perhaps optimally) large. After a triangulation is performed, each point is a node for roughly one to three dozen tetrahedra. The tetrahedra typically number slightly greater than six times the number of points.

This triangulation is done at the beginning of each time step, with no connectivity information saved from the preceding.

With this triangulation, the integral in (2) over the volume of the rotational flow becomes a sum of integrals over each of the tetrahedral volumes. An $\mathrm{O}\left(\mathrm{N}^{2}\right)$ operation is avoided by implementing an 'acceleration' algorithm which reduces the variation in computational time with the number of points to $\mathrm{O}(\mathrm{N} \log \mathrm{N})[15,16]$. The points are grouped into boxes containing an approximately equal number of points, and (2) is evaluated for the integration volume of the appropriate boxes via a multipole expansion. The integrals to compute the terms in the multipole expansion are performed with the aid of the tetrahedra, and up to three terms are kept in the expansion.

During a velocity calculation at a particular field point, the integration of (2) is carried out with this acceleration scheme for all boxes whose associated error is less than the maximum allowed. Of the remaining tetrahedra, most are evaluated numerically using Gaussian quadrature [17]. The error associated with the quadrature is found using a multipole expansion of the integrand in (2); we assume linear variation of the vorticity within the respective tetrahedra. For a given field point, most of the remaining (typically over 90 percent) tetrahedra have a 1-point quadrature error less than the maximum allowed. Of those tetrhedra remaining after these 1-point quadratures are performed, most have a 5-point quadrature error less than the maximum allowed. Finally, roughly two dozen tetrahedra remain which are too close to the field point to be treated numerically. The integration over these is outlined in [18]: the vorticity over each of these tetrahedra is taken to be the average of that at the four nodes of each tetrahedron and taken out of the integral (2), the resulting volume integral is converted by integration by parts to a surface integral over the triangular faces of the tetrahedra, and these four integrals are computed using the expressions provided in [19].

\section{Computation of the velocity derivatives}

The velocity gradient tensor is computed by a moving least-squares method [20]. On the neighbors about each point a quadratic polynomial is fit the the values of the field variable (in this case each component of velocity) to be differentiated. The constant term in the polynomial is taken to be the value of the field variable at the point in question, and the coefficients multiplying the nine terms of the polynomial are specified by the fit. Nine equations are obtained by minimizing the sum over the neighbor points of the square of the difference between the polynomial and the variable. These constants and thus the first and second derivatives of the polynomial are found by solution of the $9 \times 9$ set at each point. The field 
variable derivatives are taken to be those of the polynomial. For each point, the set of neighboring points is provided by the triangulation; for computer codes not employing Delaunay triangulation, other schemes can provide this set.

Although the moving least-squares method has proven to be a useful tool for computing derivatives, there are two features which should be noted. One is that the algorithm is not conservative. The second is that, although possible, an implicit timestepping algorithm based on the method is too complicated to be feasible.

When viscous flows are computed, application of this method to the vorticity field provides the required Laplacians.

\section{Adaptive refinement of the set of computational points}

From this discussion of the integration and differentiation algorithms, their accuracy depends on the accuracy of the interpolation within each tetrahedron (for integration) or over the neighborhood (for differentiation). The following scheme is designed to maintain the interpolation accuracy and hence the accuracy of the calculation. At the beginning of each time step, for each tetrahedron, the difference between the interpolated values found from the moving least-square method applied to the vorticity magnitude at each of the four points and the actual vorticity at the other three points, respectively, is examined. If the maximum of this difference divided by the average vorticity magnitude in the tetrahedron is greater than an allowed value, a new point is added at the centroid of the tetrahedron with vorticity on the new point set to the average of the four nodes. For the calculations, presented below the maximun allowed value is 0.2 .

\section{Computational Results}

\section{$\underline{\text { Initial conditions }}$}

We present the computational results of the inviscid interaction of two vortex rings. Two cases are examined, and these are nearly identical except for the ring radii. In the first case, the ring radius $\mathrm{R}$ is four times the core diameter of the rings, and in the second case the ring radius is two times the core diameter. The initial core radius $\sigma$ and the initial circulation $\Gamma$ are used to nondimensionalize the calculations, so that in nondimensional variables $\sigma=1$ and $\Gamma=1$ at time $\mathrm{t}=0$ in both cases. The initial vorticity magnitude $\omega$ is uniform in the cross-section of each ring, so that at $t=0, \omega=1 / \pi$. The initial planes of the rings are perpendicular to each other. For the $\mathrm{R}=2$ case, the centroids of the rings are separated by a distance of 6 , and for the $\mathrm{R}=4$ case, this distance is 8 . To define the vortex rings, the vorticity is set on 100 planes of 35 points each. To enhance the resolution of the calculation in the region of interaction, the planes are spaced so that their separation along the core axis increases from about 0.063 at the point of least initial separation of the rings to about 0.169 at the point of greatest separation for the $\mathrm{R}=2$ case, with the $\mathrm{R}=4$ case being twice these values. The $\mathrm{x}$ coordinate is defined by the direction of initial separation of the ring centroids, and the y direction is defined by the initial direction from this axis of their point of closest approach. On the $\mathrm{z}=0$ plane, the vorticity lies in the $\mathrm{z}$ direction, and it is this component that will be used to consider the dynamics of the interaction.

\section{Overview of the interaction}

The $\mathrm{R}=4$ case is discussed first. Figure 1 shows the surface of the rotational flow at the initial time and three later times. The surface is colored on the velocity magnitude, with red indicating the highest velocity, blue the lowest velocity. Also shown is the connectivity generated by the Delaunay triangulation. We see that, indeed, unlike the viscous case, no vorticity line reconnection between the 
(a)

(b)

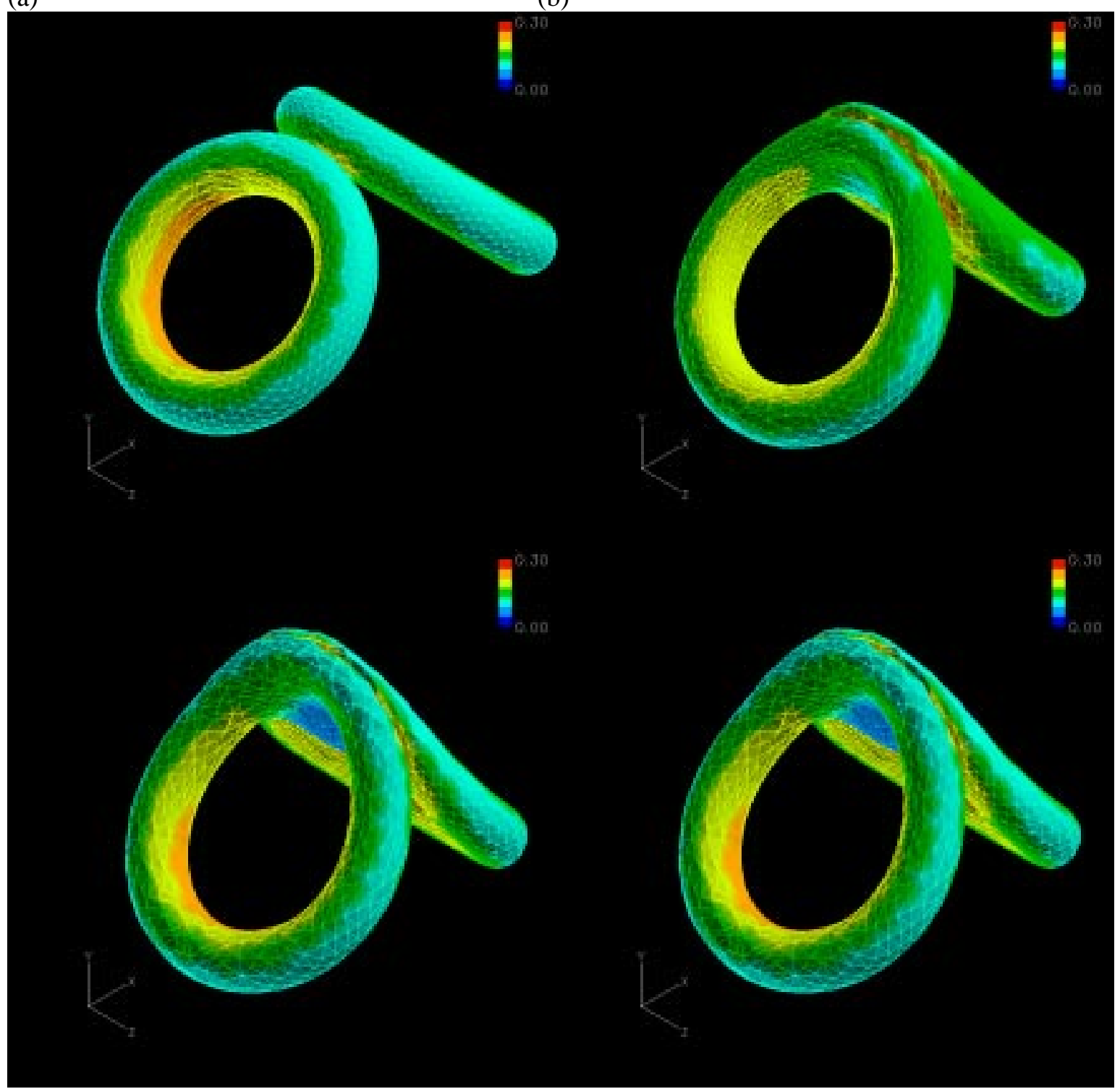

(c)

(d)

Figure 1. The surface of the rotational flow of both rings for the $\mathrm{R}=4$ case at (a) $\mathrm{t}=0$, (b) $\mathrm{t}=25.1$, (c) $\mathrm{t}=$ 34.2 , (d) $t=38.0$. The surface is colored on the velocity magnitude, with red indicating a magnitude of 0.3 or greater.

rings occurs. Instead, the vorticity where the rings collide is deformed, with the cross-section becoming strongly distorted from its original circular form. Figure 2 shows just one ring, so that the region of interaction may be seen. As the interaction proceeds, an area of high velocity develops on part of the surface of the rings nearest one another. At the same time on this surface, an adjacent region of very slow moving fluid is created. Together these two areas make up a nearly planar face on each ring, which is separated from its counterpart on the other ring by an increasingly thin layer of irrotational flow.

(a)

(b) 


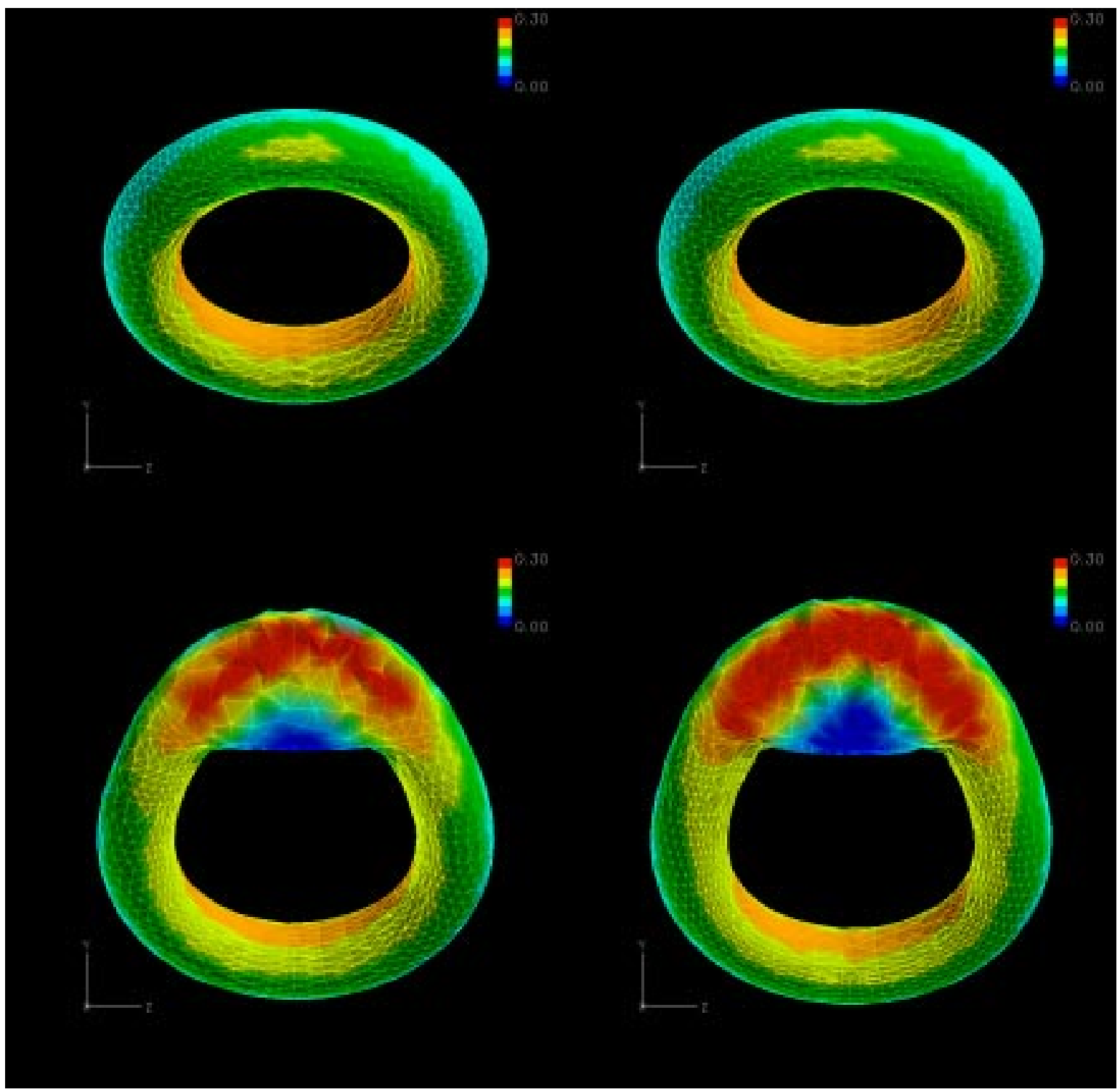

(c)

(d)

Figure 2. Another view of one of the two rings in Figure 1 at the same times as shown there.

Additional aspects of the structure of the velocity field in the neighborhood of closest approach are seen in Figure 3, and are consistent with the features seen in Figures 1 and 2 on the surface of the ring. As the interaction progresses, the cross-section of the rotational flow becomes increasingly thinner, as it must due to stretching along the out-of-plane axis. The center of rotation within each ring propagates upward, leaving behind a region of very slow moving, but still rotational, fluid. A very thin layer of relatively rapidly moving fluid develops along the interface between the centers of rotation. These thin layers are separated by a layer of irrotational flow, itself increasingly thin as the interaction proceeds.

This progressive flattening of the region of rotational flow in the neighborhood of the interaction is seen in other calculations of the inviscid interaction of vortices [e.g., Figure 3 of 8]. Additionally, also seen in other studies is the accompanying appearance of an active head and slowly moving tail in cross-sections of the rotational flow which is a prominent feature of Figure 3.

(a) 


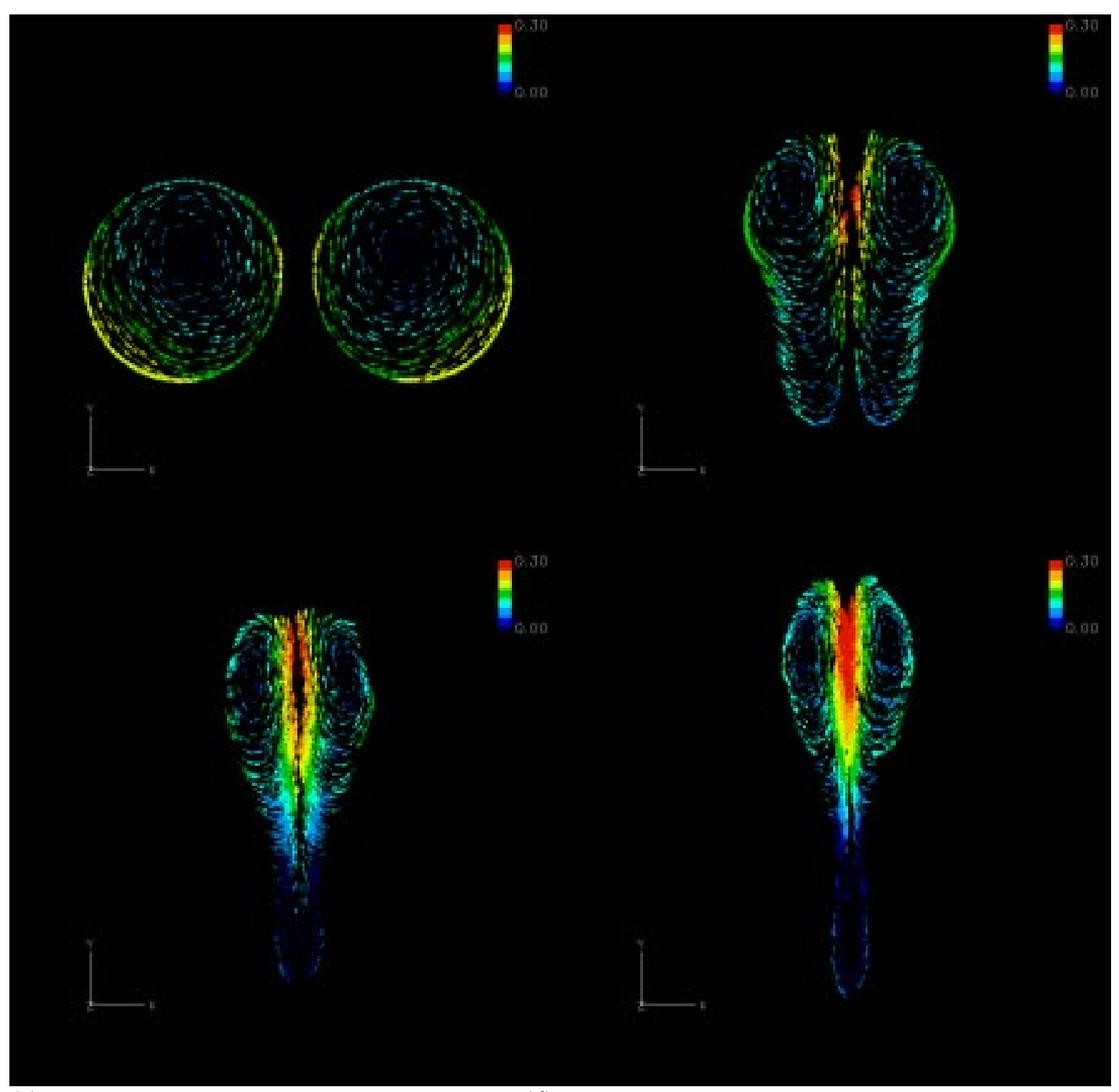

(c)

(d)

Figure 3. Velocity vectors on computational points near the plane of symmetry at the four times shown in Figure 1. The color scale is the same as in Figure 1.

The interaction of the $\mathrm{R}=2$ rings is qualitatively similar. Figure 4 shows the surface of the rotational flow for these fatter rings near the end of the calculation, and the corresponding velocity vectors in the crosssection of the rings near the plane of symmetry. The characteristic flattening of the rotational region, development of a head-and-tail structure, and intensificaton in the head region of the velocity in a thin layer where the rings are nearest are prominent features of the interaction.

In order to maintain resolution as the interaction proceeds, the algorithm described in Section 2 has increased the number of points from 7000 at $t=0$ to approximately 50,000 at the end of the calculation; the number of tetrahedra at the end is about 300,000 . 
(a)

(b)

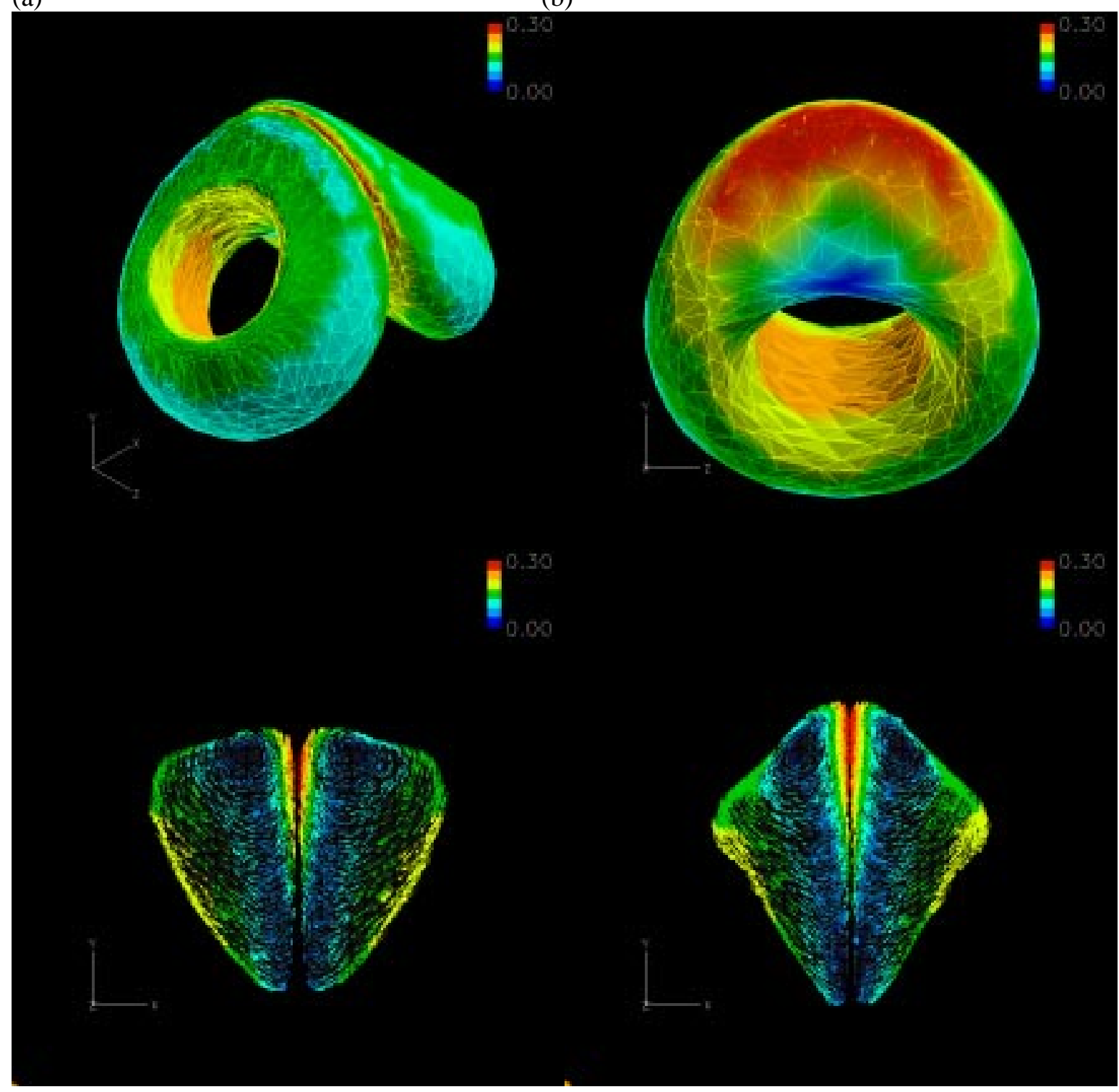

(c)

(d)

Figure 4. Four views of the $\mathrm{R}=2$ interaction. (a) The surface of both rings, colored on velocity magnitude, at $\mathrm{t}=23.4$ (red indicates a magnitude of 0.3 or greater); (b) the surface of one ring at $t=23.4$; (c) velocity vectors on computational points near the plane of symmetry at $t=20.6$ and $(d) t=23.4$. The color scale is the same on all four views.

\section{Approach to singularity?}

Since the maximum norm of the vorticity controls the breakdown of smooth solutions to the 3-D Euler equations [4], we assess the possible approach to singularity in these interactions in terms of the vorticity. As the thin layers of rapidly moving fluid develop in the head region of the interface, they are accompanied by increasingly intense sheets of vorticity lying near the surface of the rings (Figure 5). The growth of the maximum of the absolute value of the z-component of the vorticity (which in these cases is essentially the maximum of the norm of the vorticity) is shown quantitatively in Figure 6 . The difference in the curve for the respective rings in this plot is an indication of the uncertainty in the calculation due to time and space discretization in the calculation. Comparison of the $R=4$ results in Figures 5 and 6 with the $R=2$ results shows some effects of the poorer spatial resolution of the former case. 


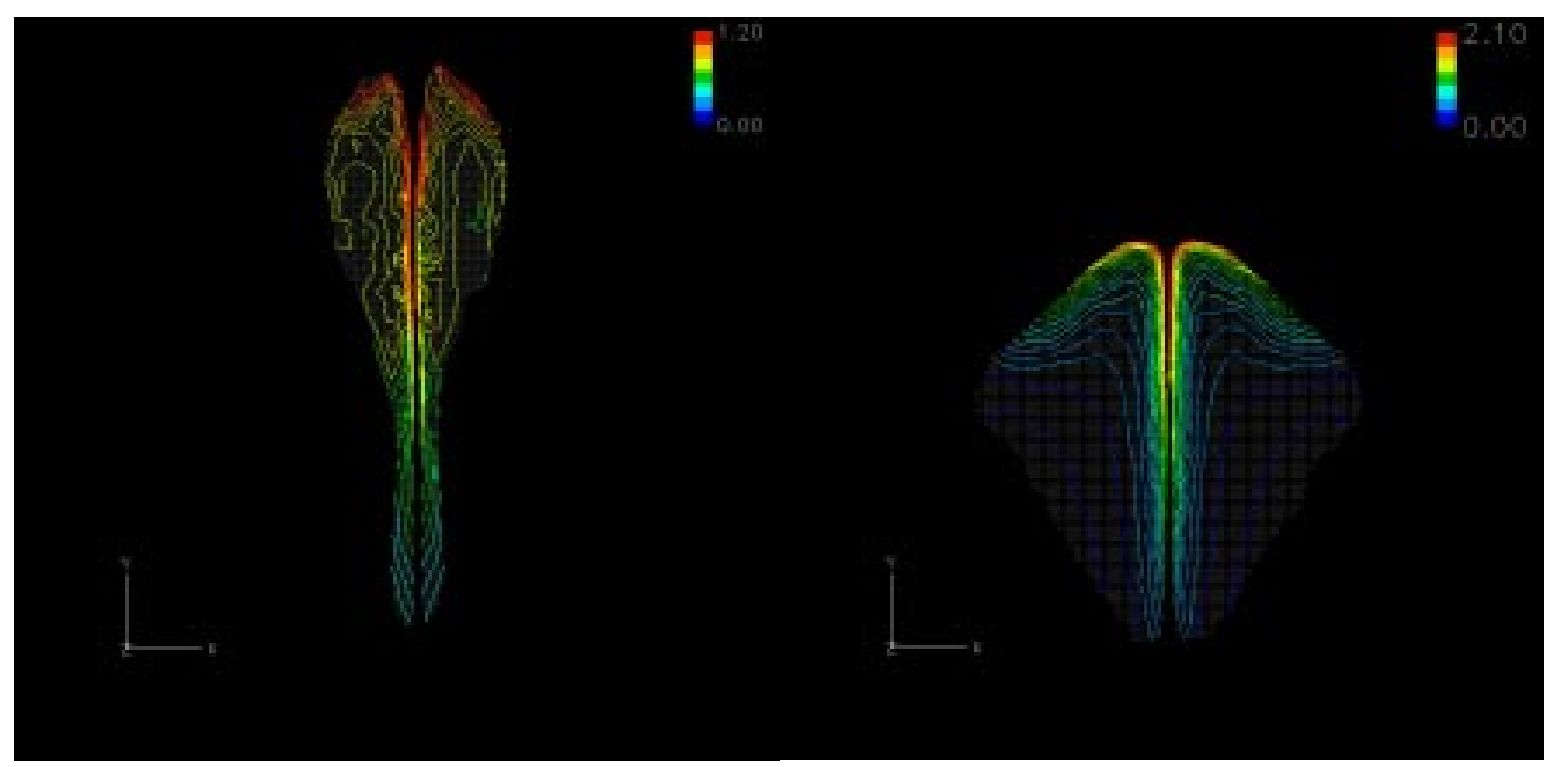

(a)

(b)

Figure 5. Contours of the z-component of vorticity lying in the plane of symmetry (the $\mathrm{z}=0 \mathrm{plane}$ ) of the interaction. (a) The $\mathrm{R}=4$ case at $\mathrm{t}=38.0$. (b) The $\mathrm{R}=2$ case at $\mathrm{t}=23.4$.

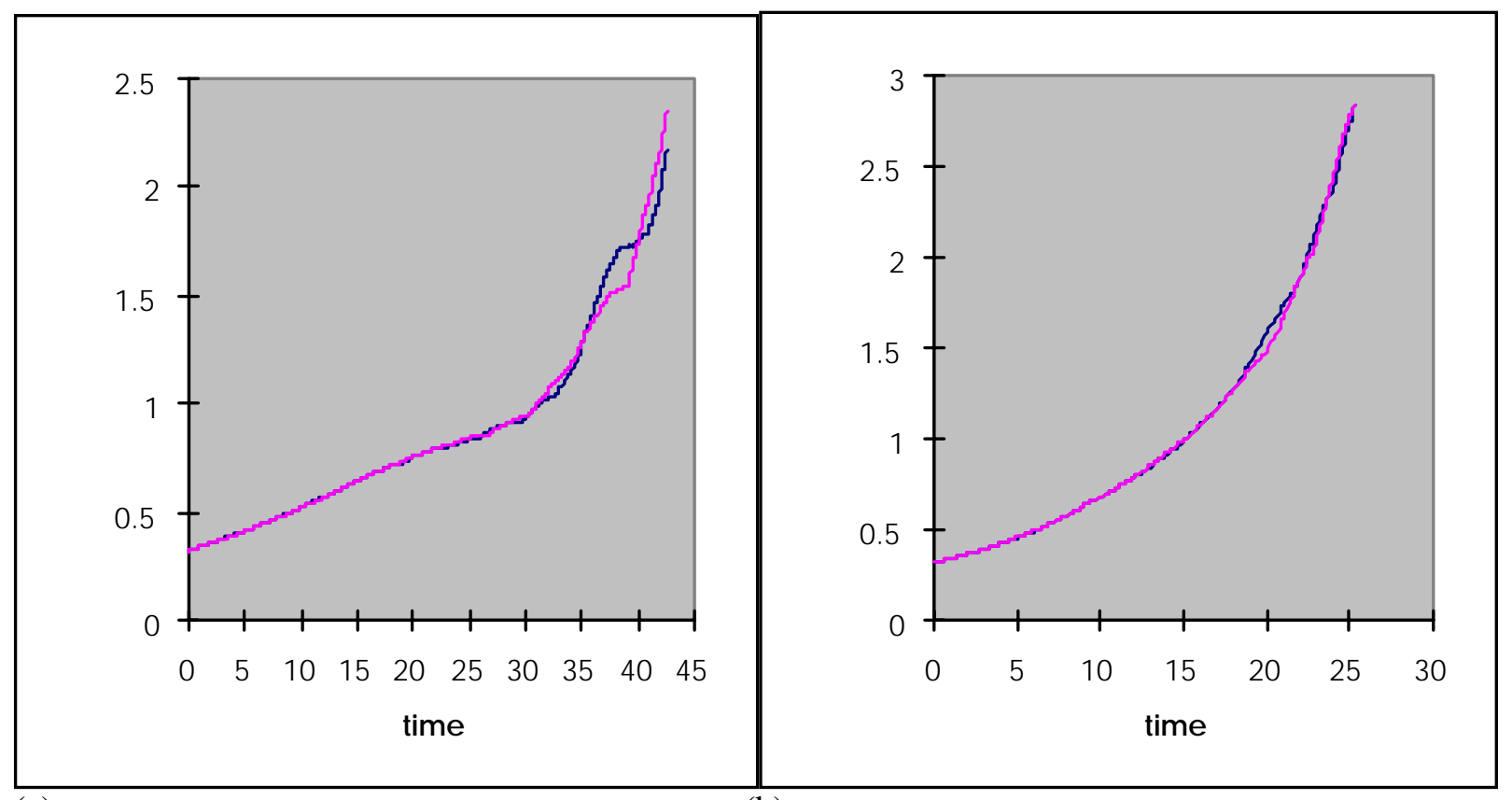

(a)

(b)

Figure 6. Maximum of the absolute value of the $\mathrm{z}$-vorticity in each ring, versus time. (a) The $\mathrm{R}=4$ case. (b) The $\mathrm{R}=2$ case.

It is anticipated $[4,8]$ that as a singularity at $t=t_{c}$ is approached the maximum norm of the vorticity behaves as $\omega=1 /\left(t_{c}-t\right)$. If a singularity is being approached in these calculations, the inverse of the $\mathrm{z}$ component of vorticity should display a linear decrease with time. On the other hand, if saturation of the stretching has occured, the maximum norm should grow exponentially. The behavior of the maximum of the absolute value of the $\mathrm{z}$-component of the vorticity in the $\mathrm{R}=2$ cases is examined by the plots shown in Figure 7. Taken together, the plots indicate that through most of the development of the interaction, the growth in this maximum is exponential; indeed, the flow is far from singularity during this time. However, 
both plots also show that a transition in the nature of the growth occurs at about $\mathrm{t}=20$. Unfortunately, in this calculation the computed interval beyond this time is too short to

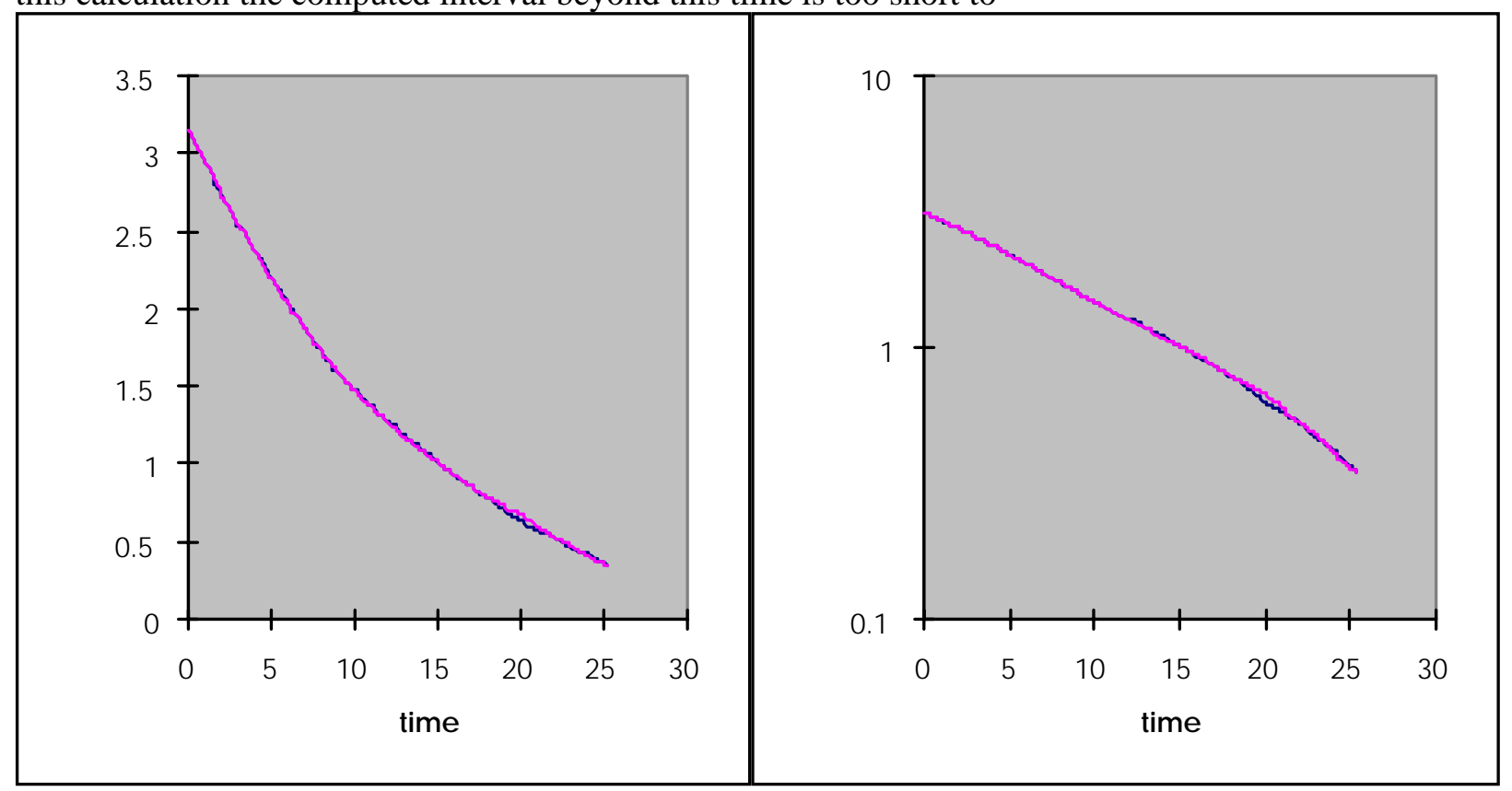

(a)

(b)

Figure 7. Inverse of the maximum of the absolute value of the z-component of vorticity versus time for the $\mathrm{R}=2$ case. (a) Linear-linear scale. (b) Log-linear scale.

definitively confirm or deny that this new flow regime is an approach to a finite-time singularity. The large area of flattened geometry of the vortex core might suggest that the flow has saturated and thus the vorticity norm will only grow exponentially. However, there is strong curvature of the head structure in the $\mathrm{z}$ direction. Kerr [8] makes the point that such curvature is an important ingredient in the emergence of a finite-time singularity. Indeed, the form of the intense sheets of vorticity on the inner faces and at the top of the contour plot for the $R=2$ case in Figure 5 bear a qualitative resemblance to Figure 4 of [8], which plots contours of vorticity near the time and location of a possible singularity.

Thus there is some motivation to pursue this calculation, particularly the $\mathrm{R}=2$ case, to later time, probably to the neighborhood of $t=30$. In this case, symmetry should be used to speed the calculation.

\section{Acknowledgments}

The research of JRG was supported by the Office of Naval Research under grant 98WX20138. The program manager is James Fein. The research of JSM was supported by the Army Research Office under grant DAAH04-96-1-0081. The program manager is Dr. Thomas L. Doligalski. Computer time was provided by the DoD Supercomputer Centers and the NUWC Advanced Scientific and Engineering Computing Center. 


\section{References}

1 Kida, S. \& M. Takaoka: Vortex reconnection. Ann. Rev. Fluid Mech. 26 (1994), 169-189,

2 Helmholtz, H.: Uber diskontinuierliche Flussigkeitsbewegungen. Monatsber. K. Akad. Wiss. Berlin 23 (1858), 215-228. See Philos. Mag 4(36) (1968), 337-346.

3 Ladyzenskaya, O.: The Mathematical Theory of Viscous Incompressible Flow. New York: Gordon \& Breach, 2nd edition (1963).

4 Beale, J.T., T. Kato \& A. Majda: Remarks on the breakdown of smooth solutions for the 3-D Euler equations. Commun. Math. Phys. 94 (1984), 61-66.

5 Constantin, P.: SIAM Rev. 36 (1994), 73.

6 Constantin,P. \& C. Fefferman: Indiana Univ. Math. J. 42 (1994), 775.

7 Bhattacharjee, A., C.S. Ng and Xiaogang Wang: Finite-time vortex singularity and Kolmogorov spectrum in a symmetric three-dimensional spiral model. Phys. Rev. E 52(5) (1995), 5110-5123.

8 Kerr, R.M.: Evidence for a singularity of the three-dimensional, incompressible Euler equations. Phys. Fluids A 5(7) (1993), 1725-1746.

9 Kerr, R.M. \& F. Hussain: Simulation of vortex reconnection. Physica D 37 (1989), 474.

10 Boratav, O.N., R.B. Pelz \& N.J. Zabusky: Reconnection in orthogonally interacting vortex tubes: Direct numerical simulations and quantification in orthogogonally interacting vortices. Phys. Fluids A 4 (1992), 581.

11 Marcus, D.L. \& J.B. Bell: Vorticity intensification and transition to turbulence in the three-dimensional Euler equations. Commun. Math. Phys. 147 (1992), 371.

12 Press, W.H., S.A. Teukolsky, W.T. Vetterling and B.P. Flannery: Numerical Recipes. New York: Cambridge University Press (1992).

13 de Berg, M., M. van Kreveld, M. Overmars \& O. Schwarzkopf: Computational Geometry, Heidelberg: Springer-Verlag (1997)

14 Borouchaki, H. \& S.H. Lo: Fast Delaunay triangulation in three dimensions. Comput. Methods Appl. Mech. Engng 128 (1995), 153-167.

15 Greengaard, L. \& V. Rokhlin: A fast algorithm for particle simulations. J. Comp. Phys. 73 (1987), 325248.

16 Strickland, J.H. \& R.S. Baty: An overview of fast multipole methods. Sandia National Laboratories Report SAND95-2405, November, 1995.

17 Zienkiewicz, O.: The Finite Element Method. New York: McGraw-Hill (1977) p. 202.

18 Grant, J.R., S.A. Huyer \& J.S. Uhlman: Solution of the vorticity equation on a Lagrangian mesh using triangularization: computation of the Biot-Savart integral in three-dimensions. Forum on Vortex Methods for Engineering Applications Papers. Sandia National Laboratory, Albuquerque, NM. February, 1995.

19 Newman, J.N.: Distributions of sources and normal dipoles over a quadrilateral panel. J. Engineering Math. 20 (1986), 113-126. 
20 Marshall, J.S. \& J.R. Grant: A Lagrangian vorticity collocation method for viscous, axisymmetric flows with and without swirl. J. Comp. Phys. 136 (1997), 302-330. 